\title{
Diagnosis and Management of UTI in Febrile Infants Age 0-2 Months: Applicability of the AAP Guideline
}

\author{
Pearl W Chang, MD*, Marie E Wang, MD, MPH², Alan R Schroeder, MD²
}

'Department of Pediatrics, Seattle Children's Hospital / University of Washington, Seattle, Washington; ${ }^{2}$ Department of Pediatrics, Stanford University School of Medicine, Stanford, California.

Urinary tract infections (UTIs) are the most common bacterial infection in young infants. The American Academy of Pediatrics' (AAP) clinical practice guideline for UTIs focuses on febrile children age 2-24 months, with no guideline for infants $<2$ months of age, an age group commonly encountered by pediatric hospitalists. In this review, we assess the applicability of the AAP UTI Guideline's action statements for previously healthy, febrile infants $<2$ months of age. We also discuss additional considerations in this age group, including concurrent bacteremia and routine testing for meningitis. Journal of Hospital Medicine 2020;15:176180. () 2020 Society of Hospital Medicine $\bigcup$ rinary tract infections (UTIs) are the most common bacterial infection and one of the most common reasons for hospitalization in young infants. ${ }^{1,2}$ The American Academy of Pediatrics (AAP) has published several clinical practice guidelines for the evaluation and management of febrile children ages 2-24 months with first-time UTIs, most recently in 2011 and affirmed in $2016 .{ }^{3}$ These guidelines do not provide recommendations for infants aged $<2$ months, which leads to uncertainty regarding the diagnosis and management of UTIs for infants in this age group. We assess the applicability of the AAP UTI Guideline's action statements for infants aged $<2$ months presenting with first-time UTIs, with an emphasis on recent evidence. Because the considerations for bacterial infections differ for febrile infants aged $<2$ months compared with older infants, we do not discuss action statements one and two (determination of the likelihood of UTIs and decision to test urine) and statement seven (medical evaluation for fever after first UTI). ${ }^{3}$ Additionally, because concomitant bacteremia and meningitis are more common in this age group than in older infants, we review some of the controversies surrounding the diagnosis and treatment of these disease entities.

\section{DIAGNOSIS}

"Action Statement 3: To establish the diagnosis of UTI, clinicians should require both urinalysis results that suggest infection (pyuria and/or bacteriuria) and the presence of at least 50,000 colony-forming units (CFUs) per $\mathrm{mL}$ of a uropathogen cultured from a urine specimen obtained through catheterization or SPA." ${ }^{3}$

\footnotetext{
*Corresponding Author: Pearl Chang, MD; E-mail: pearlchangmd@gmail.com; Telephone: 206-987-8099; Twitter: @pearlchang.

Published online first February 19, 2020
}

Received: August 30, 2019; Revised: October 19, 2019;

Accepted: October 30, 2019

๑ 2020 Society of Hospital Medicine DOI 10.12788/jhm.3349
To distinguish asymptomatic bacteriuria or contamination from a true UTI, the AAP Guideline requires both a positive urinalysis (UA) and culture for a diagnosis of a UTI. ${ }^{3}$ Historically, the UA was considered to be poorly sensitive for infections in young infants, with older studies reporting sensitivities ranging from $40 \%$ to $82 \%$ using urine culture as the gold standard. ${ }^{4-7}$ Thus, infants aged $<2$ months with positive urine cultures and negative UAs are often treated as having true UTIs, though this practice varies by institution. ${ }^{8}$ Possible explanations for the low UA sensitivity in this population include rapid bladder emptying, immature immune systems, and inability to concentrate urine. However, a negative UA plus a positive urine culture could also represent a "true negative" UA and a "false positive" culture, a finding that may be more common in young infants in whom sterile urine obtainment is often challenging.

Two recent studies have addressed this issue by evaluating the UA sensitivity in patients with bacteremic UTIs, as growth of the same pathogenic organism from the blood and urine almost certainly represents true infection. ${ }^{9,10}$ In a retrospective study of 203 infants aged $<3$ months with bacteremic UTIs, the presence of any leukocyte esterase (LE) or pyuria ( $>3$ white blood cells per high-powered field [WBC/HPF]) had a sensitivity of $99.5 \%$ (95\% Cl: $98.5 \%-100 \%)$ and specificity of $93.9 \%$ (95\% Cl: $87.8 \%-93.2 \%) .{ }^{9}$ In a prospective, multicenter study of 4,147 febrile infants aged $\leq 60$ days, of whom 27 infants had bacteremic UTIs, a positive UA (any LE, $>5 \mathrm{WBC} / \mathrm{HPF}$, or nitrite) had a sensitivity and specificity of 1.00 (95\% Cl: 0.87-1.00) and 0.91 (95\% Cl: 0.90-0.91), respectively. ${ }^{10}$ Although screening tests may appear to have higher sensitivity in more severely diseased populations ("spectrum bias"), ${ }^{11}$ it is not clear that infants with bacteremic UTIs are definitively sicker than infants with nonbacteremic UTIs (see "bacteremic UTI" section below). Additionally, this study found similarly excellent sensitivity (0.94 [95\% Cl: 0.90-0.96]) and specificity (0.91 [95\% Cl: 0.90-0.91]) of the UA among infants with nonbacteremic UTIs, including infants $<28$ days old. ${ }^{10}$

UA sensitivity (using urine culture as the gold standard) may 
be lower for non-Escherichia coli UTIs. ${ }^{9,10,12}$ In a retrospective study that included 90 infants $<2$ months old with UTIs, urine cultures yielding Pseudomonas aeruginosa, Enterococcus, or Klebsiella species were significantly less likely (odds ratio [95\% Cl]: 0.19 [0.06-0.60]; 0.14 [0.07-0.28]; 0.34 [0.17-0.68], respectively) to have pyuria ( $\geq 5$ WBC/HPF) or LE (1+ or greater) than urine cultures yielding E. coli., ${ }^{12}$ though an alternative explanation for this finding is that these organisms may be more likely to cause asymptomatic bacteriuria or contamination. ${ }^{13}$

The appropriate CFU/mL threshold to define a UTI and the extent that this threshold should vary by urine collection methods are still unclear. In the aforementioned bacteremic UTI study, 12 patients with E. coli bacteremia had urine cultures with $<50,000 \mathrm{CFU} / \mathrm{mL}$ plus pyuria (WBC or LE) in the UA, indicating that true UTIs may occur with $<50,000 \mathrm{CFU} / \mathrm{mL}$.

Based on these recent studies, we believe that the recommendation to incorporate UA results into the diagnoses of UTIs can be applied to infants $<2$ months old, as well as consideration for a UTI for colony counts of $\geq 10,000 \mathrm{CFU} / \mathrm{mL}$ if the UA is positive. For infants with positive urine cultures and negative UAs who have not received antibiotics, we suggest repeating both studies if treatment is being considered. For those who have started antibiotics, the pretest probability of a UTI, initial illness severity, and risks and benefits of continuing treatment should be considered.

\section{TREATMENT}

"Action Statement 4a: When initiating treatment, the clinician should base the choice of route of administration on practical considerations. Initiating treatment orally or parenterally is equally efficacious. The clinician should base the choice of agent on local antimicrobial sensitivity patterns (if available) and should adjust the choice according to sensitivity testing of the isolated uropathogen. ${ }^{\text {"3 }}$

Most infants <2 months old with UTIs are hospitalized initially because of fever. Therefore, the decision point for most clinicians is not whether to hospitalize but for how long to hospitalize and treat with intravenous (IV) antibiotics prior to discharging home on oral antibiotics. Although all-oral antibiotic regimens are used to treat UTIs in older infants and children, ${ }^{14-18}$ to our knowledge, there are no randomized controlled trials (RCTs) comparing all-IV vs all-oral antibiotics or a longer vs shorter initial IV course that include infants $<1$ month old. In the trials that do include infants aged 1-2 months, ${ }^{14,18}$ the number of subjects in this age group is too small to draw conclusions, a finding supported by a 2014 Cochrane review. ${ }^{19}$ An adequately powered RCT of different IV antibiotic durations in this age group would be challenging. For example, nearly 1,000 subjects would be needed to demonstrate a statistically significant difference between a $5 \%$ and $10 \%$ relapse risk between groups, a difference that some may find clinically important.

The paucity of evidence in this age group may explain the considerable variability in the approach to IV antibiotic duration in young infants. Concerns about enteral absorption and underdeveloped immune systems may prompt some physi- cians to treat the youngest patients more aggressively. One study demonstrated that the proportion of patients $<2$ months old receiving prolonged courses ( $\geq 4$ days) of IV antibiotics for UTIs in 46 U.S. children's hospitals ranged from $0 \%$ to $67 \%{ }^{20}$ Similar variability across hospitals has been described in other observational studies ${ }^{21,22}$ and across subspecialties in one survey of pediatricians. ${ }^{23}$

Several observational studies provide additional evidence supporting shorter IV courses. In two studies that examined administrative databases, there was no difference in treatment failure rates between infants aged $<2$ months $^{20}$ and $<6$ months ${ }^{21}$ receiving longer ( $\geq 4$ days) vs shorter IV courses. In a study of 172 infants $<1$ month old with UTIs, the median IV duration was 4 days (range 2-12 days), and no subjects experienced treatment failure or relapse. ${ }^{24}$ In a multicenter study of 251 infants $<3$ months old with bacteremic UTIs, mean IV antibiotic durations ranged from 5.5-12 days, and no patient had a relapsed bacteremic UTI. Six infants $(2.4 \%)$ had a relapsed UTI without bacteremia, with no association between IV antibiotic duration and relapse. ${ }^{22}$

Based on the available data and known risks of hospitalization and prolonged IV therapy, a reasonable approach for infants $<1$ month old would be to hospitalize for two to three days while awaiting blood and cerebral spinal fluid (CSF) culture results. Given the possibility of Enterococcus or Enterobacteriaceae that are resistant to third-generation cephalosporins, standard therapy of ampicillin and gentamicin for febrile neonates is reasonable, assuming there is no concern for meningitis. Antibiotics should be narrowed when susceptibilities are known. Once culture results return and signs and symptoms have resolved, discharge home on oral antibiotics is justifiable based on the available literature. For well-appearing infants aged 1-2 months with a presumptive UTI (based on UA results), if hospitalization is not warranted for other reasons, then we recommend outpatient treatment with oral or intramuscular therapy based on local susceptibilities (typically a cephalosporin) and close follow-up for one to two days while awaiting culture results. Although empiric cephalosporin therapy may not provide $100 \%$ coverage for all potential organisms, clinical deterioration is uncommon in infants and children receiving discordant therapy. ${ }^{25}$

"Action Statement 4b: The clinician should choose 7 to 14 days as the duration of antimicrobial therapy."

The AAP's recommendation to provide antibiotics (by oral or parenteral route) for a minimum of seven days total stems from a 2002 meta-analysis comparing long (7-14 days) vs short ( $\leq 3$ days) courses, where the pooled relative risk of treatment failure with short-course therapy was 1.94 (95\% Cl: 1.19-3.15). ${ }^{26}$ However, in this analysis, the trials that demonstrated inferiority with short courses were all trials that used single doses of antibiotics, and a similar Cochrane review comparing 2-4 days with 7-14 days demonstrated no differences in outcomes. ${ }^{27}$ Therefore, shorter total courses, but not a single dose, are probably appropriate for most UTIs in children. Although there are no obvious biologic reasons why longer total courses would be needed in young infants, there are unfortunately 
limited data comparing different total antibiotic durations in this age group. We believe that 7-14 days of total therapy is a reasonable recommendation for infants $<2$ months old, and that future studies should investigate shorter total courses.

\section{IMAGING}

"Action Statement 5: Febrile infants with UTIs should undergo renal and bladder ultrasonography (RBUS)." ${ }^{3}$

The AAP Guideline acknowledges that the RBUS is a poor screening test for the detection of genitourinary abnormalities in infants. ${ }^{3}$ The RBUS can be normal in infants with vesicoureteral reflux (VUR) or show nonspecific findings of unclear clinical significance. ${ }^{28}$ In a prospective study of 220 infants $<3$ months old by Tsai et al, 9/39 infants (23\%) with grade III-V VUR had normal RBUS. ${ }^{29}$ Studies that included older infants have found a similar false-negative rate of $0 \%-40 \%$ for detecting grade IV-V VUR by RBUS. ${ }^{28}$ Nonetheless, since a RBUS is safe and noninvasive, we feel that the benefits of screening for abnormalities such as hydronephrosis (that could indicate posterior urethral valves or ureteropelvic junction obstruction) outweigh the risks (eg, false positives, overdiagnosis, and cost) of performing a RBUS after a first-time UTI.

"Action Statement 6a: Voiding cystourethrography (VCUG) should not be performed routinely after the first febrile UTI; VCUG is indicated if RBUS reveals hydronephrosis, scarring, or other findings that would suggest either high-grade VUR or obstructive uropathy, as well as in other atypical or complex clinical circumstances." 3

"Action Statement 6b: Further evaluation should be conducted if there is a recurrence of febrile UTI."3

The RBUS may be normal in infants with VUR. Therefore, the AAP's recommendation to perform a VCUG only if the RBUS is abnormal or after a recurrent UTI concedes that there will be infants with VUR who are missed after the first UTI. ${ }^{3}$

The United Kingdom's National Institute for Health and Care Excellence guideline recommends a VCUG for infants $<6$ months old with a bacteremic or non-E. coli UTI. ${ }^{30}$ Whether high-grade VUR is more common in young infants with bacteremic UTIs than nonbacteremic UTIs remains inconclusive. In the Honkinen et al. study that included 87 infants $<3$ months old with bacteremic UTIs, the prevalence of grade IV-V VUR (10\%) and obstruction (7\%) was higher than that of the 88 nonbacteremic infants (2\% grade IV-V VUR and $2 \%$ with obstruction). In the multicenter study of 251 infants $<3$ months old with bacteremic UTIs, the prevalence of grade IV-V VUR was $12.1 \% .{ }^{31}$ This is higher than that of the nonbacteremic infants in Honkinen et al.'s study 32 but more similar to the prevalence of grade IV-V VUR found in Tsai et al. (8.2\%) and Ismaili et al.'s $(7.0 \%)$ studies of UTIs in general. ${ }^{29,33}$

There does appear to be a higher prevalence of urinary tract abnormalities in young infants with non-E. coli vs $E$. coli UTIs. ${ }^{31,32,34,35}$ The odds of an abnormal VCUG was $8.0(95 \% \mathrm{Cl}$ : 2.3-28) times higher for non-E. coli than E. coli UTIs in the study of 251 bacteremic infants. ${ }^{31}$ In a study of 122 infants $<3$ months old, the odds of grade III-V VUR was 10 (95\% Cl 2.6-41) times higher for non-E. coli than E. coli UTIs. ${ }^{35}$
However, the need for early detection of VUR is controversial, and VCUGs are invasive, involve ionizing radiation, and may require sedation. Two recent trials (one which included only children with VUR and another in which $42 \%$ of subjects had VUR) demonstrated a modest effect of prophylactic antibiotics in preventing recurrent UTIs ( $>5,000$ doses of antibiotics needed to prevent one UTI recurrence), but the effect size did not differ by the presence or degree of VUR, and neither demonstrated any benefit in reducing future renal scarring. ${ }^{36,37}$ The benefit of surgical interventions for VUR also remains unclear, though studies are limited. ${ }^{38}$ Overall, there is no evidence suggesting that infants $<2$ months old require more vigilance for VUR detection than the 2-24 month age group.

\section{SPECIAL CONSIDERATIONS}

\section{Bacteremic UTI}

The prevalence of bacteremia in infants $\leq 60$ days old with UTIs was $9 \%$ in a study conducted from 2008 to 2013 in 26 EDs and has ranged from $3 \%$ to $17 \%$ in older studies. ${ }^{10,} 22$ Many studies have described similar clinical and laboratory findings in young infants with bacteremic and nonbacteremic UTIs. ${ }^{39-41}$ Despite this, bacteremic UTIs have been associated with prolonged parenteral antibiotic courses, resulting in longer hospitalizations and increased costs. ${ }^{40}$ Two recent multicenter studies of infants with bacteremic UTIs ( 251 infants $<3$ months old ${ }^{22}$ and 115 infants $\leq 60$ days old ${ }^{42}$ ) demonstrated variable IV courses and no association between IV duration and relapsed UTI. The latter study showed no risk difference in the adjusted 30-day UTI recurrence (risk difference $3 \%, 95 \% \mathrm{Cl}$ : -5.8 to 12.7 ) or allcause reutilization (risk difference $3 \%, 95 \% \mathrm{Cl}$ : -14.5 to 20.6 ) between long and short IV groups. ${ }^{42}$ Neither study had patients with relapsed bacteremic UTIs or reported that patients suffered clinical deterioration while on oral antibiotics. ${ }^{22,42}$

Based on these data demonstrating that adverse outcomes are rare in infants with bacteremic UTIs and not associated with parenteral antibiotic duration, we recommend short parenteral courses (2-3 days) with conversion to oral therapy once infants have clinically improved.

\section{Positive Urinalysis and Testing for Meningitis}

Multiple risk stratification algorithms for febrile infants aged $\leq 60$ days categorize infants with a positive UA (and therefore likely UTI) as high-risk for having concomitant bacteremia or meningitis, for which lumbar puncture (LP) is typically recommended. ${ }^{43-45}$ The risk of not testing CSF is the potential to insufficiently treat meningitis because treatment for UTIs and meningitis differ in dosing, route, and duration. Recent studies have challenged the practice of routine LPs for infants aged 1-2 months with a suspected UTI due to the low prevalence (0\%-0.3\%) of concomitant meningitis. ${ }^{39,46-48} \mathrm{~A}$ meta-analysis of 20 studies reporting rates of concomitant meningitis with UTI in infants aged $29-90$ days found a pooled prevalence of $0.25 \%$ (95\% Cl: $0.09 \%-0.70 \%) .{ }^{49}$ Furthermore, a study of febrile infants ages 29-60 days found that the prevalence of meningitis did not differ between those with a positive vs negative UA (3/337 [0.9\%] vs $5 / 498$ [1.0\%], respectively), suggesting that a positive 
UA alone should not modify the pretest probability of meningitis in this age group. ${ }^{50}$

Two studies have also examined the risk of delayed meningitis among infants $\leq 60$ days old treated for UTIs without CSF testing. A northern California study that examined 345 episodes among 341 UA-positive infants aged 29-60 days found zero cases (95\% Cl: 0\%-1.1\%) of delayed meningitis within 30 days of evaluation..$^{50} \mathrm{~A}$ multicenter study of well-appearing febrile infants aged 7-60 days found 0/505 cases $(95 \% \mathrm{Cl}$ : 0\%$0.6 \%$ ) of delayed meningitis within 7 days of discharge; 407 (81\%) were aged $31-60$ days..$^{51}$ In summary, studies have shown a low rate of concomitant meningitis and a low risk of delayed meningitis in infants aged 1-2 months treated for UTI without CSF testing. Given this, clinically targeted (eg, based on ill appearance and/or lethargy), rather than routine, CSF testing in this age group can be considered.

\section{CONCLUSION}

While the AAP UTI Guideline is directed toward 2-24-monthold infants, recent evidence suggests that action statements 3-6 apply to infants $<2$ months old. Incorporation of pyuria as a diagnostic criterion for UTIs, early transition to oral therapy, and selective VCUG testing are all warranted based on the available evidence and consideration of known risks and benefits. Future studies with larger sample sizes that include infants $<2$ months old would be beneficial to ensure that the available studies, which have relatively small cohorts, do not suffer from type II error. We propose that future studies examine shorter ( $<7$ days) vs longer total antibiotic duration, shorter vs longer initial IV antibiotics (especially in infants $<1$ month old or with bacteremic UTIs), and whether RBUS can be performed in a targeted manner. RCTs comparing universal vs targeted imaging strategies would help ascertain whether the increased diagnostic yield that accompanies more aggressive imaging strategies translates into improved outcomes. Application of these AAP guidelines to the $<2$-month age group and enhancement of the evidence base can promote the high-value care of young infants with UTIs.

Disclosures: Dr. Chang and Dr. Wang have no conflicts of interest disclosures. Dr. Schroeder has received honoraria for grand rounds presentations on the subject of urinary tract infections.

\section{References}

1. Greenhow TL, Hung YY, Herz AM, Losada E, Pantell RH. The changing epidemiology of serious bacterial infections in young infants. Pediatr Infect Dis J. 2014;33(6):595-599. https://doi.org/10.1097/INF.0000000000000225

2. Spencer JD, Schwaderer A, McHugh K, Hains DS. Pediatric urinary tract infections: an analysis of hospitalizations, charges, and costs in the USA. Pediatr Nephrol. 2010;25(12):2469-2475. https://doi.org/10.1007/s00467-010$1625-8$

3. Subcommittee On Urinary Tract Infection. Reaffirmation of AAP Clinical Practice Guideline: the diagnosis and management of the initial urinary tract in fection in febrile infants and young children 2-24 months of age. Pediatrics. 2016;138(6):1-5. https://doi.org/10.1542/peds.2016-3026.

4. Crain EF, Gershel JC. Urinary tract infections in febrile infants younger than 8 weeks of age. Pediatrics. 1990;86(3):363-367. https://doi.org/10.1542/ peds.105.2.e20

5. Dayan PS, Bennett J, Best $R$, et al. Test characteristics of the urine Gram stain in infants $<$ or $=60$ days of age with fever. Pediatr Emerg Care. 2002;18(1):1214. https://doi.org/10.1097/00006565-200202000-00004.

6. Bachur R, Harper MB. Reliability of the urinalysis for predicting urinary tract infections in young febrile children. Arch Pediatr Adolesc Med. 2001;155(1):6065. https://doi.org/10.1001/archpedi.155.1.60.

7. Reardon JM, Carstairs KL, Rudinsky SL, Simon LV, Riffenburgh RH, Tanen DA. Urinalysis is not reliable to detect a urinary tract infection in febrile infants presenting to the ED. Am J Emerg Med. 2009;27(8):930-932. https://doi. org/10.1016/j.ajem.2008.07.015

8. Schroeder AR, Lucas BP, Garber MD, McCulloh RJ, Joshi-Patel AA, Biondi EA. Negative urinalyses in febrile infants age 7 to 60 days treated for urinary tract infection. J Hosp Med. 2019;14(2):101-104. https://doi.org/10.12788/ jhm.3120.

9. Schroeder AR, Chang PW, Shen MW, Biondi EA, Greenhow TL. Diagnostic accuracy of the urinalysis for urinary tract infection in infants $<3$ months of age. Pediatrics. 2015;135(6):965-971. https://doi.org/10.1542/peds.20150012.

10. Tzimenatos L, Mahajan P, Dayan PS, et al. Accuracy of the urinalysis for urinary tract infections in febrile infants 60 days and younger. Pediatrics. 2018;141(2):e20173068. https://doi.org/10.1542/peds.2017-3068.

11. Newman TB, Kohn MA. Evidence-based diagnosis. Practical Guides to Biostatistics and Epidemiology. Cambridge; New York: Cambridge University Press, 2009

12. Shaikh N, Shope TR, Hoberman A, Vigliotti A, Kurs-Lasky M, Martin JM. Association Between Uropathogen and Pyuria. Pediatrics. 2016;138(1):e20160087. https://doi.org/10.1542/peds.2016-0087.

13. Eliacik K, Kanik A, Yavascan $O$, et al. A comparison of bladder catheterization and suprapubic aspiration methods for urine sample collection from infants with a suspected urinary tract infection. Clin Pediatr. 2016;55(9):819-824. https://doi.org/10.1177/0009922815608278.

14. Bocquet N, Sergent Alaoui A, Jais JP, et al. Randomized trial of oral versus sequential IV/oral antibiotic for acute pyelonephritis in children. Pediatrics. 2012;129(2):e269-e275. https://doi.org/10.1542/peds.2011-0814.

15. Bouissou F, Munzer C, Decramer S, et al. Prospective, randomized trial comparing short and long intravenous antibiotic treatment of acute pyelonephritis in children: dimercaptosuccinic acid scintigraphic evaluation at 9 months. Pediatrics. 2008;121(3):e553-e560. https://doi.org/10.1542/peds.2006-3632.

16. Hodson EM, Willis NS, Craig JC. Antibiotics for acute pyelonephritis in children. Cochrane Database Syst Rev. 2007(4):CD003772. https://doi. org/10.1002/14651858.CD003772.pub3.

17. Neuhaus TJ, Berger C, Buechner K, et al. Randomised trial of oral versus sequential intravenous/oral cephalosporins in children with pyelonephritis. Eur J Pediatr. 2008;167(9):1037-1047. https://doi.org/10.1007/s00431-007-0638-1

18. Hoberman A, Wald ER, Hickey RW, et al. Oral versus initial intravenous therapy for urinary tract infections in young febrile children. Pediatrics. 1999;104(1 Pt 1):79-86. https://doi.org/10.1542/peds.104.1.79.

19. Strohmeier Y, Hodson EM, Willis NS, Webster AC, Craig JC. Antibiotics for acute pyelonephritis in children. Cochrane Database Syst Rev. 2014(7):CD003772. https://doi.org/10.1002/14651858.CD003772.pub4.

20. Lewis-de Los Angeles WW, Thurm C, Hersh AL, et al. Trends in intravenous antibiotic duration for urinary tract infections in young infants. Pediatrics. 2017;140(6):e20171021. https://doi.org/10.1542/peds.2017-1021.

21. Brady PW, Conway PH, Goudie A. Length of intravenous antibiotic therapy and treatment failure in infants with urinary tract infections. Pediatrics. 2010;126(2):196-203. https://doi.org/10.1542/peds.2009-2948.

22. Schroeder AR, Shen MW, Biondi EA, et al. Bacteraemic urinary tract infection: management and outcomes in young infants. Arch Dis Child. 2016;101(2):125-130. https://doi.org/10.1136/archdischild-2014-307997.

23. Joshi NS, Lucas BP, Schroeder AR. Physician preferences surrounding urinary tract infection management in neonates. Hosp Pediatr. 2018:8(1):21-27. https://doi.org/10.1542/hpeds.2017-0082.

24. Magin EC, Garcia-Garcia JJ, Sert SZ, Giralt AG, Cubells CL. Efficacy of short-term intravenous antibiotic in neonates with urinary tract infection. Pediatr Emerg Care. 2007;23(2):83-86. https://doi.org/10.1097/PEC. 0b013e3180302c47

25. Wang ME, Lee V, Greenhow TL, et al. Clinical response to discordant therapy in third-generation cephalosporin-resistant UTIs. Pediatrics. 2019; In press.

26. Keren R, Chan E. A meta-analysis of randomized, controlled trials comparing short- and long-course antibiotic therapy for urinary tract infections in children. Pediatrics. 2002;109(5):E70. https://doi.org/10.1542/peds.109.5.e70.

27. Michael M, Hodson EM, Craig JC, Martin S, Moyer VA. Short versus standard duration oral antibiotic therapy for acute urinary tract infection in children. Cochrane Database Syst Rev. 2003(1):CD003966. https://doi. org/10.1002/14651858.CD003966. 
28. Finnell SM, Carroll AE, Downs SM, Subcommittee on Urinary Tract I. Technical report-Diagnosis and management of an initial UTI in febrile infants and young children. Pediatrics. 2011;128(3):e749-e770. https://doi.org/10.1542/ peds.2011-1332.

29. Tsai JD, Huang CT, Lin PY, et al. Screening high-grade vesicoureteral reflux in young infants with a febrile urinary tract infection. Pediatr Nephrol. 2012;27(6):955-963. https://doi.org/10.1007/s00467-012-2104-1.

30. National Institue for Health and Care Excellence. Urinary Tract Infection in Children. http://www.nice.org.uk/guidance/cg54/evidence/cg54-urinarytract-infection-in-children-full-guideline2. Published August 2007. Accessed August 2019

31. Chang PW, Abidari JM, Shen MW, et al. Urinary imaging findings in young infants with bacteremic urinary tract infection. Hosp Pediatr. 2016;6(11):647652. https://doi.org/10.1542/hpeds.2015-0229.

32. Honkinen O, Jahnukainen T, Mertsola J, Eskola J, Ruuskanen O. Bacteremic urinary tract infection in children. Pediatr Infect Dis J. 2000;19(7):630-634. https://doi.org/10.1097/00006454-200007000-00009

33. Ismaili K, Lolin K, Damry N, Alexander M, Lepage $P$, Hall M. Febrile urinary tract infections in 0- to 3-month-old infants: a prospective follow-up study. J Pediatr. 2011;158(1):91-94. https://doi.org/10.1016/j.jpeds.2010.06.053.

34. Cleper R, Krause I, Eisenstein B, Davidovits M. Prevalence of vesicoureter al reflux in neonatal urinary tract infection. Clin Pediatr. 2004;43(7):619-625. https://doi.org/10.1177/000992280404300706.

35. Pauchard JY, Chehade H, Kies CZ, Girardin E, Cachat F, Gehri M. Avoidance of voiding cystourethrography in infants younger than 3 months with Escherichia coli urinary tract infection and normal renal ultrasound. Arch Dis Child. 2017;102(9):804-808. https://doi.org/10.1136/archdischild-2016-311587.

36. Craig JC, Simpson JM, Williams GJ, et al. Antibiotic prophylaxis and recurrent urinary tract infection in children. N Engl J Med. 2009;361(18):1748-1759. https://doi.org/10.1056/NEJMoa0902295.

37. Hoberman A, Greenfield SP, Mattoo TK, et al. Antimicrobial prophylaxis for children with vesicoureteral reflux. N Engl J Med. 2014;370(25):2367-2376. https://doi.org/10.1056/NEJMoa1401811.

38. Williams G, Hodson EM, Craig JC. Interventions for primary vesicoureteric reflux. Cochrane Database Syst Rev. 2019;(2):CD001532. https://doi. org/10.1002/14651858.CD001532.pub4

39. Schnadower D, Kuppermann N, Macias CG, et al. Febrile infants with urinary tract infections at very low risk for adverse events and bacteremia. Pediatrics. 2010;126(6):1074-1083. https://doi.org/10.1542/peds.2010-0479,

40. Roman HK, Chang PW, Schroeder AR. Diagnosis and management of bac- teremic urinary tract infection in infants. Hosp Pediatr. 2015;5(1):1-8. https:// doi.org/10.1542/hpeds.2014-0051.

41. Newman TB, Bernzweig JA, Takayama JI, Finch SA, Wasserman RC, Pantell $\mathrm{RH}$. Urine testing and urinary tract infections in febrile infants seen in office settings: the Pediatric Research in Office Settings' Febrile Infant Study. Arch Pediatr Adolesc Med. 2002;156(1):44-54. https://doi.org/10.1001/archpedi.156.1.44.

42. Desai $\mathrm{S}$, Aronson PL, Shabanova $\mathrm{V}$, et al. Parenteral antibiotic therapy duration in young infants with bacteremic urinary tract infections. Pediatrics. 2019;144(3):e20183844. https://doi.org/10.1542/peds.2018-3844,

43. Gomez B, Mintegi S, Bressan S, et al. Validation of the "Step-by-Step" approach in the management of young febrile infants. Pediatrics. 2016;138(2):e20154381. https://doi.org/10.1542/peds.2015-4381.

44. Kuppermann N, Dayan PS, Levine DA, et al. A clinical prediction rule to identify febrile infants 60 days and younger at low risk for serious bacterial infections. JAMA Pediatr. 2019;173(4):342-351. https://doi.org/10.1001/jamapediatrics.2018.5501.

45. DePorre AG, Aronson PL, McCulloh RJ. Facing the ongoing challenge of the febrile young infant. Crit Care. 2017;21(1):68. https://doi.org/10.1186/s13054017-1646-9,

46. Tebruegge M, Pantazidou A, Clifford V, et al. The age-related risk of co-existing meningitis in children with urinary tract infection. PLoS One. 2011;6(11):e26576. https://doi.org/10.1371/journal.pone.0026576.

47. Thomson J, Cruz AT, Nigrovic LE, et al. Concomitant bacterial meningitis in infants with urinary tract infection. Pediatr Infect Dis J. 2017;36(9):908-910. https://doi.org/10.1097/INF.0000000000001626.

48. Wallace SS, Brown DN, Cruz AT. Prevalence of concomitant acute bacterial meningitis in neonates with febrile urinary tract infection: a retrospective cross-sectional study. J Pediatr. 2017;184:199-203. https://doi.org/10.1016/j. jpeds.2017.01.022.

49. Nugent J, Childers M, Singh-Miller N, Howard R, Allard R, Eberly M. Risk of meningitis in infants aged 29 to 90 days with urinary tract infection: a systematic review and meta-analysis. J Pediatr. 2019;212:102-110.e5. https://doi. org/10.1016/j.jpeds.2019.04.053.

50. Young BR, Nguyen THP, Alabaster A, Greenhow TL. The prevalence of bacterial meningitis in febrile infants $29-60$ days with positive urinalysis. Hosp Pediatr. 2018;8(8):450-457. https://doi.org/10.1542/hpeds.2017-0254.

51. Wang ME, Biondi EA, McCulloh RJ, et al. Testing for meningitis in febrile well-appearing young infants with a positive urinalysis. Pediatrics. 2019;144(3):e20183979. https://doi.org/10.1542/peds.2018-3979. 